\title{
Las aulas de clase: espacios para crear posibilidades ${ }^{1}$
}

\author{
Yenny Lucila Daza Vidarte ${ }^{2}$
}

\begin{abstract}
Resumen
Esta propuesta busca crear espacios de reflexión en donde maestros e intelectuales como referentes de realidad puedan aportar a la construcción de proyectos de vida, humanidad, sentido, re-significación de la educación y tejido social desde las aulas de clase.

Ya que las aulas de clase son el espacio pertinente para formar resistencia en busca de horizontes de mundo y humanidad, en donde el educando se reconozca desde sí, encontrando significado y sentido a la educación, teniendo en cuenta su contexto, sus recursos y contingencias, donde maestros e intelectuales desde la articulación de su discurso y su proceder puedan brindar las posibilidades de cambio y de esta manera poder repensar la escuela en pro de emancipar la educación.
\end{abstract}

Palabras clave: aulas de clase, proyectos de vida, maestros e intelectuales, humanidad, tejido social, sentido.

\section{Classrooms: Spaces to create possibilities}

\begin{abstract}
This proposal seeks to create spaces for reflection in which teachers and scholars as reality references may contribute to the construction of the project of life, humanity, respect, education re-signification and social fabric from the classroom.

Due to classrooms are the appropriate space to form resistance searching for the world and humanity horizons, where the student is recognized from himself, finding meaning and sense to education, taking into account its context, resources and contingencies, where teachers and intellectuals from the articulation of speech and their actions may provide the potential for change and rethink the school concept in favor of the emancipating education.

Keywords: Classrooms, project of life, teachers and intellectuals, humanity, social fabric, sense
\end{abstract}

1 Recibido: 13 de julio de 2012. Aceptado: 21 de agosto de 2012.

2 Yenny Lucila Daza Vidarte. Docente e investigadora de instituciones educativas en Neiva, investigadora dentro del Macroproyecto Maestros e intelectuales en Colombia, Licenciada en Educación Infantil Integrada. Magister en Educación Docencia. Correo electrónico: yeya629@hotmail.com. 


\section{Las aulas de clase: espacios para crear posibilidades}

\author{
De pronto el porvenir existía; \\ me transformaría en otra, qué \\ diría yo, y ya no sería yo. \\ Beauvoir Simone
}

La educación es importante y fundamental en el desarrollo del ser humano, ya que la tarea de enseñar es como dice Quintar (1998, 15):

Bella por lo de humano, ética por su hacer, compleja por esa misma humanidad que compromete cada acto, cada opción qué, como, quién y para que enseñar, en la conciencia de la proyección que esto tiene en la transformación colectiva de quienes aprende-enseña, enseñan - aprenden, recuperando el valor revolucionario de los microespacios que configuran los macroespacios sociales, dimensiones que hacen de la enseñanza un proceso trascendente en las propias circunstancias.

Ante esto la enseñanza cobra un valor fundamental en los espacios sociales, es un aporte al sujeto para que éste potencie desde sí y hacía la colectividad, entonces, si la educación es tan importante, ¿Por qué lo reducimos a la transmisión de una serie de contenidos cerrados en un currículo?, ¿Por qué se sigue en la idea de dejar de lado la subjetividad, creatividad del estudiante en la construcción del currículo? ¿Por qué ha de ser más fácil dejar de crear posibilidades de mundo desde el aula y dedicarse a trasmitir contenidos? ¿Será que la profesión de ser docente ha dejado su belleza de lo humano? ¿Será que el sujeto dejo de ser el centro de la educación, para y por quién se enseña? Estos interrogantes llevan a considerar la idea de repensar la educación, como el espacio pertinente para una construcción en colectivo, desde el aporte de maestros e intelectuales, creando un espacio de diá- logo y transformación de los sujetos. Entonces ahora Como señala "el desafío es desnaturalizar lo que se es y lo que se vive para generar, como acto educativo, actos de con-ciencia fundados científicamente y promover la libertad como "capacidad de elección” (Quintar, 1998, 28).

Quizás es el momento de cuestionarnos frente a las circunstancias que se viven en la enseñanza -aprendizaje y su efecto que deja en las prácticas sociales, ya que el ejercicio de la docencia tiene una obligación frente a la sociedad y es la de la contribuir cada día a la construcción de una mejor educación para que ésta aporte a la personalidad de los estudiantes y a la búsqueda de horizontes en colectividad. Es que no se puede seguir atrapado en la idea que se puede hacer mucho desde la transmisión de contenidos; es importante comenzar ahora y restablecer la idea que lo substancial en la educación, es el sujeto, la humanidad, porque desde allí, desde la interacción con el otro, desde el aporte del otro y la construcción en colectivo es donde se pueden generar los horizontes de mundo, la potenciación de construcción de proyectos vitales.

Es importante retomar la escuela como un espacio donde se construye posibilidades desde la dialógicidad, desde lo cotidiano, en donde hay un cruce de saberes (docentes, estudiantes, padres de familia), en donde se debe tener en cuenta el conocimiento del entorno, de los ecosistemas, en donde juega un papel las tecnologías ya que ellas son el medio de comunicación más utilizado por niños, niñas y jóvenes, en donde cada día el docente revise su "equipaje", en donde "Las dinámicas del sujeto se articule con las propias del contexto" (Méndez, 2009, 189) construyendo en colectividad.

Se debe rescatar en la escuela, la calidad humana como valor agregado, a través de la condición humana, desde las interrelaciones sociales, dejando de lado la idea errónea que si el sistema no proporciona los recursos, entonces, desde 
el aula ya no se puede crear posibilidades de mundo, es importante que el maestro e intelectual retome como ser político el interés que tenía frente a la educación como actor participativo desde su actuar y no sólo como un generador de ideas desde la escritura, ya que el aula de clases puede ser un espacio conveniente para crear las posibilidades de aprendizaje, de oportunidades para potenciar los proyectos de vida. Es allí donde se puede generar el cambio, pues en esos espacios todos los autores entran a participar desde sus vivencias, intereses, es allí donde se puede crear un punto de encuentro entre los diferentes mundos que son las personas.

Por esa razón los maestros e intelectuales son los llamados a aportarle a la educación a ser generadores de cambio y es allí en la aulas de clase donde se debe formar la resistencia para el cambio, buscando la construcción de humanidad y tejido social; el maestro e intelectual debe enfrentarse a ese "sistema de poder que obstaculiza, que prohíbe, que invalida ese discurso ese saber" (los intelectuales y el poder) deben enfrentarlos desde la pluma que utilizan para "poner en letras los pensamientos de la comunidad" (Entrevista, Dinvisky, 2012) ${ }^{3}$ y desde su práctica como sujeto social y político que es.

\section{Inicio del camino}

El desarrollo global ha colocado al sujeto en una relación inevitable con la realidad externa que lo separa de si mismo, es ahora un reto recuperarse como sujeto en su propia singularidad, la educación es un agente importante en este reconocimiento, por esa razón los actores que allí participan deben actuar articuladamente para que la escuela sea un espacio en donde se evidencia la belleza de lo humano.

3 Dinvisky Daniel. (2012). Sin pensamiento crítico. En http://cultura.elpais.com/cultura/2012/02/22/ actualidad/1329922234_215883.html (Recuperado el 07 de julio del 2012 )
Es de reconocer que la educación tiene una estructura curricular que debe seguir, pero esta no debe ser construida ni trabajada sin tener en cuenta a los sujetos, en especial la subjetividad de los estudiantes, pues son ellos los ejes centrales de la educación.

Por consiguiente se hace necesario pensar en un rescate de valores que humanice pero no desde la perspectiva de repetir o registrar en los cuadernos la importancia del respeto, la tolerancia o de la solidaridad, no. Estos valores deben ir más allá, dicho valores corresponde a la idea de permitir un análisis reflexivo frente a la construcción de proyectos vitales que desvinculen de los sujetos la incapacidad de reconocer posibilidades de mundo desde su contexto.

En donde desde la autonomía de la persona se pueda construir posibilidades, desde sus recursos, desde su contexto, desde sus intereses pero como dice Zemelman (2007) "Idea de autonomía y construcción que se corresponde con pensar desde valores que den cuenta de lo humano sin agotarlo en la conformación de sus determinaciones sociales".

Ya que el sujeto como ser social, en este caso los maestros e intelectuales, están dados a relacionarse y desde allí como se relacione, motive o potencie desde el aula será un agente participativo encaminado a crear posibilidades de cambio, disminuyendo el academismo que muchas veces no permite flexibilidad en las prácticas pedagógicas haciendo que los sujetos inmersos en ellas sientan que cumplen un "ritual escolar" generando una brecha discursiva entre la comunidad, padres, estudiantes y llevando a un sin sentido, negando las posibilidades de construcción que a su vez genera una desarticulación entre las mismas.

Todo esto generando un declive en la educación, ya que no se da un trabajo en colectividad ocasionando de esta manera una perdida de identidad, de construcción, por esa razón es significativo que desde 
las aulas de clase se generen espacios de reconocimiento de los sujetos, en donde cada uno cumpla el papel importante que le corresponde en esa construcción de humanidad y tejido social.

$Y$ de esta manera la escuela no sé comprendería solo desde una lógica de contenidos disciplinares, sino desde el significado que pueda tener. La educación es una construcción de significado, el significado requiere que el sujeto lo construya y no que esté inscrito, por esa razón es importante pensar en colocar la ciencia en contexto, lo que posibilitaría al estudiante construir sentido para su propia vida desde el aula de clase, permitiéndole al educando crear posibilidades desde sus interese, desde su subjetividad en donde se encuentren rutas de mundo y genere un tejido social.

Desde esa perspectiva el aula sería un espacio de transformación en donde el estudiante se dejaría de ver como alguien que recibe información y se empezaría a ver como un sujeto con interese y posibilidades, de esta manera se iniciaría otra visión, permitiendo la oportunidad de crear programas que dinamicen el reconocimiento de sí mismo, de su identidad y que desde este reconozca al otro y se articule a las dinámicas de su contextos desde sus recursos, desde sus posibilidades buscando "la capacidad del sujeto para desplegar su siendo en el contexto de realidades en proceso de cambio"(Zemelman, 2007, 9-10)

Cambios que podrían realizarse desde diferentes puntos de vista como el conocimiento pleno de los ecosistemas, pues estos generan identidad, un reconocimiento de historicidad como sujeto, lo que aportaría a un afirmación del apoyo de las generaciones anteriores, creando una re-significación, generando espacios de construcción en colectividad, porque "la educación, en realidad, ha de centrarse en la relación entre el sujeto y el mundo que lo acoge". (Meirieu, 1998, 70) Forjando humanidad, pues muchas veces el desconocimiento del legado de las generaciones anteriores produce falta de identidad, arraigo y confianza.

Esa falta de historicidad, de memoria, ese olvido por lo que las generaciones pasadas contribuyeron a la construcción de tejido social, demuestra una necesidad de cohesión social en donde el sujeto reconozca al otro como un agente importante en su propia construcción de subjetividad, pues está "es una construcción individual, atravesada por la colectividad, por lo que construyo de mi tanto soy con el otro" (Gil, $2009,45)$ esto daría un punto de partida para esa transformación cultural humana.

Por esa razón es elemental que cada día el maestro revise su "equipaje" pues como sujeto movilizador es el primero en reconocerse así mismo desde sí para que realice un aporte significativo a sus estudiantes como referente de vida, es necesario que esté en constante introspección pues lo maestros e intelectuales son los llamados a crear espacios de movilización y construcción en colectividad. Especialmente el maestro que es quién constantemente en la cotidianidad del aula se relaciona con el estudiante y desde allí en su coexistir cada día tiene la oportunidad de descubrir nuevas formas de vivir, enseñar, de relacionarse con el otro, con el entorno, de construir humanidad.

En consecuencia maestros e intelectuales tiene como función permitirle a los educandos construirse a sí mismo como dice Meirieu (1998):

Sujeto en el mundo, heredero de una historia en la que sepa que está en juego, capaz de comprender el presente y de inventar el futuro es por ello que partiendo de sus historias y vivencias se podría crear desde el aula de clase un espacios de reflexión buscando significados y sentidos de mundo como referentes de realidad que contribuya a los proyectos de vida y horizontes de mundo. 
Es importante que el maestro e intelectual inicie una construcción de significado desde su actuar frente a la crisis de humanidad que se vive desde la escuela, desde las aulas que son espacios en donde se puede crear mayor resistencia, ya que la globalización crea sistemas que muchas veces obstaculizan la re-significación de la educación, de horizontes, de vida, de humanidad.

Por esa razón la escuela, desde sus aulas esta llamada a formar sujetos críticos que aporten al cambio desde el reconocimiento de sí mismo, teniendo en cuenta al otro y su contexto; sujetos que no se dejen arrastrar por lo aparente o que masifica.

\section{Humanidad}

En la actualidad las personas viven corriendo por todos lados, pues esta modernidad acelerada los impulsa a estar con afanes, pendientes de horarios en el trabajo, en el colegio de los hijos, en el momento de ir a pagar los recibos, todo en busca de capitalizar para un mejor estatus social, o tan solo para poder sobrevivir cada día, "en las calles hombres y mujeres apresurados avanzan sin mirarse pendientes de cumplir horarios que hacen peligrar su humanidad" (Sábato, 2000,121).

Peligra la humanidad en esta sociedad, ya las familias no tiene tiempo de compartir, ya esos momentos en el comedor en donde se platicaba sobre los sucesos de la mañana, o se compartían circunstancias felices o tal vez tristes buscando apoyo de algún miembro de la familia fue relegado a tan solo estar sentados como autómatas pendientes del nuevo "miembro de la familia" el televisor, que ha robado un gran espacio en ese compartir, en esa construcción de humanidad, ya el diálogo paso a ser tan solo una palabra que se utiliza para contestar a la pregunta ¿como resuelven en tu casa los problemas? Pues se habla poco entre padres e hijos, ya solo es un comunicar o informar en la familia utilizando los Blackberrys ya que es más sencillo enviar un mensaje diciendo... hoy no llego a almorzar, mamá me demoro me invitaron a cine. Y no es que la tecnología sea la causante de la deshumanización, es el uso inadecuado que le dan las personas.

Cada día la humanidad entra en crisis, pues no se hace un alto en donde exista una reflexión frente al proceder, como dice Sabato, el desarrollo de la nación necesita de atributos espirituales, de los cuales se fomenta desde la unidad familiar porque desde el rol de la familia se aporta a la construcción de una sociedad con identidad, valores, sueños y deseos de un horizonte en donde todos tengan posibilidades de humanidad. Pero esto no es solo responsabilidad de las familias ya que la escuela juega un papel importante en esta transformación social en donde desde los microespacios se aporta a los macroespacios sociales, pues "la búsqueda de una vida humana debe comenzar por la educación" (Sábato, 2000, 79)

En consecuencia la educación tiene una gran responsabilidad frente a la construcción de tejido social, de proyectos de vida y de humanidad, pues la escuela es el espacio en donde los maestros e intelectuales que como seres políticos, sociales y culturales se enfrenta a un compromiso social frente al cambio, son quiénes crean espacios de reflexión y resistencia frente a esta crisis que se vive.

Desafortunadamente en la educación pareciera que esto no tuviera relevancia pues los objetivos de la educación están bajo una lógica del sistema social actual desde un reproducción ideológica del capital cultural, "la educación no esta independizada del poder, y por lo tanto, encauza su tarea hacia la formación de gente adecuadas a las demandas del sistema" (Sábato, 2000, 81) en donde se preocupan por crear mano de obra barata y no personas criticas, reflexivas para su construcción de proyectos vitales y sentido de humanidad. 
También se evidencia que la cultura académica, sigue una lógica donde generan proyectos anclados en la racionalidad, de alguna manera censurando y dejando sin piso la posibilidad de ser, además al no hacer parte de las lógicas del sistema social y escolar se daría la idea que todos los proyectos de vida son proscritos, solo por no seguir en la misma dirección de los sistemas ahí establecidos, negándole al educando posibilidades "no tanto de construir, sino de reconocer sus opciones y momentos precisos en que son un desafío para la reflexión" (Zemelman, 1998, 16) reflexión que aportaría a la construcción de tejido social y humanidad.

Sumado a lo anterior se evidencia que en la escuela se da la reproducción ideológica clasista que se mantiene por economicismo capital y las lógicas del sistema social dominante, también por el hecho del elitismo intelectual como dice Bourdieu y Passeron "por cuanto resguarda la reproducción tanto de estructuras sociales como el de la cultura dominante" impulsando aún más la pérdida de sentido y esperanzas imposibilitando la oportunidad de crear espacios donde los educandos puedan potenciar posibilidades de nuevos horizonte.

En consecuencia la escuela busca solo una reproducción desde la trasmisión de contenidos, olvidando que "enseñar no es transferir conocimiento, sino crear las posibilidades de su producción o construcción", (Freire, 1997, 24) por esa razón muchas veces olvida la subjetividad de los educandos creando un currículo que gira bajo lógicas disciplinares sin tener en cuenta el significado que desde allí se puede construir, es cuando olvida la importancia de cruce de saberes. Porque cuando hay una participación activa de todos los actores de la comunidad educativa, padres, docentes, estudiantes, se puede trabajar en la búsqueda de una construcción colectiva y así no se enfocaría desde el individualismo que solo permite seguir deshumanizando, pues el otro no tomaría importancia dentro del constructo educativo y social.
Ahora bien si no se trabaja en colectividad, si no se tiene en cuenta al otro, si no se comparte con el otro, si no se escucha al otro, no se puede hacer una tensión entre dichas realidades, pues es necesario que los sujetos se integren entre sí y con sus entorno, de esta manera las relaciones personales, la interacción social jugaría un papel importante en la construcción de humanidad, pues ya no se pensaría desde el individualismo, sino que se haría en colectividad.

Por esa razón es importante las relaciones humanas, la interacción social, pues estas son la oportunidad de compartir con el otro, sus experiencias, vivencias y valores y de esa manera ampliar nuestros conocimientos. Pero lastimosamente esto no se da de la forma más adecuada, es evidente observar en el común la falta de tolerancia y aún más en las aulas de clase en donde los educandos se enfrentan entre sí, sin justificaciones algunas, solo por el hecho de agredir al otro ya sea de forma física, verbal y algunas veces de forma psicológica.

Esta agresión entre pares lleva un ambiente pesado de trabajo que imposibilita la oportunidad de crear un espacio reflexivo en donde el afecto este ahí pero que este se articule con relación al contexto, intereses de los educandos, teniendo en cuenta una estructura teórica que aporte a la movilización de pensamiento y aprendizaje "la interacción social es una variable esencial del desarrollo y aprendizaje, para que el funcionamiento individual es precedido y acompañado por una función interindividual" (Pontecorvo, 1993, 190).

En consecuencia este nivel de intolerancia marca una pauta de alerta, ya que esos comportamientos derriban la oportunidad en la que el sujeto se vuelva constructor de su contexto y de su proyecto de vida

"lo creado y la necesidad de creación, aquello que viene arrastrando al pasado y lo que representa una discontinuidad, un salto es lo que obliga a considerar 
a la razón como la síntesis de fatalidad y negación, inercia e interrupción de la misma" (Zemelman, 1998, 14). Por eso es normal cuestionarse sobre porque los jóvenes se comportan de determinada forma y muestran ciertas actitudes frente a los acontecimientos de su vida cotidiana y en las aulas, ya que la forma como se comporta el uno con el otro aporta a la construcción de cultura y si se sigue evidenciando intolerancia que será de las generaciones venideras, como dice Sábato (2001) "la cultura se trasmite a través de todas las actividades del hombre"

Entonces si la cultura humana sigue en ese rumbo, la sociedad tendrá una perdida incalculable que solo será valorada cuando esta ya no este, cuando el anhelo de tenerla sea más grande que la realidad de volverla alcanzar como dice Sábato (2000) "si esto no se contrabalancea con una educación que muestre lo que está pasando $y$, a la vez, promueva al desarrollo de las facultades que están deteriorándose, lo perdido será el ser humano".

Por esa razón la educación es la indicada a aportar hacia ese cambio, a mejorar las relaciones interpersonales, hacia una cultura humana, porque es allí en donde las aulas se convierten en espacios que aportarían a la transformación desde la construcción colectiva, en donde todos los actores se convertirían en agentes participativos contribuyendo al cambio desde sus contextos, desde la realidad que viven a diario.

Todo esto desde la condición humana ya que "la condición humana de la labor es la misma vida". (Arendt, 1993, 21) Y esta nunca finaliza pues está en constante construcción.

\section{Maestros e intelectuales escribir y actuar}

"El papel del intelectual no es el de situarse "un poco en avance o un poco al margen" para decir la muda verdad de todos; es ante todo luchar contra las formas de poder allí donde éste es a la vez el objeto y el instrumento: en el orden del "saber", de la "verdad", de la "conciencia", "del discurso" (Foucault, 1992, 78) el intelectual tiene un compromiso ante la sociedad como ser político, social y cultural frente al cambio, pero dicha lucha debe ser articulada entre el discurso y el actuar.

Pues no se tiene que ver al intelectual de hoy en día como un teórico, que solo buscan escribir buenos discurso o plantear ideas que la gran mayoría de veces quedan solo plasmados en el papel, aunque esto es representativo en la lucha frente al sistema y todo lo que obstaculiza, no debe ser la única manera de lucha, pues esta no tiene el mismo efecto, si no va acompañada del actuar, es importante que la resistencia frente a las circunstancias que dificultan el desarrollo y construcción de sentido y significado se de; desde las letras y el proceder.

Por esa razón es importante que el maestro e intelectual se descentralice porque algunas veces pareciera que el poder objetivo de ellos se ha silenciado por los políticos, los poderes capitalistas, los medios de comunicación, grandes mafioso, entre otros. Pero aunque no se dejen contaminar por estos grandes poderes ya no se hace resistencia como antes, solo se calla, ya no alzan su voz, su pensamiento crítico, y menos se evidencia su actuar, tan solo se hace al lado como si estuviera enfermo de apatía.

Un ejemplo de esa apatía es que maestros e intelectuales prefieren tomar distancia por los asuntos sociales, culturales, económicos, de la problemática que se vive, frente a la violencia e intolerancia, frente al sin sentido de muchos educando hacia la educación, frente a esta deshumanización.

Quizás por esta razón, esto viene manteniendo un perfil bajo, sobre todo desde la crisis dejando el territorio del pensamiento en manos de los economistas. Actualmente su misión ha sido ocupada de alguna 
manera por los indignados que no plantean su rebeldía desde un riguroso análisis intelectual sino desde lo visceral de sus experiencias. (Entrevista, Corral, 2012) ${ }^{4}$.

El maestro e intelectual es importante en la educación pues son agentes movilizadores llamados a aportar al cambio desde sus vivencias, desde sus saber, desde su proceder, pues los problemas que se evidencian en al escuela no son técnicos, son problemas en donde no se comprende la dinámica del sujeto en articulación con la dinámica del entorno, en donde no se tiene en cuenta la subjetividad, entonces es preciso crear un espacio en donde juegue un papel importante el entorno en la transformación, en donde los proyectos vitales sean trabajados desde el sujeto en construcción colectiva y para la sociedad, en donde la humanidad se construya desde las aulas de clase.

Pero para esto es importante tener en cuenta que la lectura de mundo de los maestros e intelectuales no puede ser impuesta, ni tampoco que calle lo que ha construido desde su experiencia, pero si se debe construir una relación entre su palabra y su actuar y desde allí intervenir como dice Foucault (1992): "como condición indispensable para la tarea", lo que nos lleva a reconocer que los maestros e intelectuales son importantes en el proceso educativo.

Por esa razón son ellos los llamados a crear desde las aulas de clase espacios de reflexión que aporten a la construcción de horizontes de mundo y re-significación de la educación.

\section{Posibilidades frente al sistema}

A través de la historia la educación se ha venido re-estructurando con el objetivo de brindar a los educandos una educa-

4 Corral Milagros. (2012). Sin pensamiento crítico. En http://cultura.elpais.com/cultura/2012/02/22/ actualidad/1329922234_215883.html (Recuperado el 07 de julio del 2012) ción de calidad. Un ejemplo de esta reestructuración es la revolución educativa del año 2003, que buscando proporcionar a la comunidad estudiantil una educación con calidad, construye una base bajo tres indicadores como lo son, eficiencia, cobertura y calidad.

Bajo esto indicadores inicio el proceso educativo una búsqueda hacia una nueva perspectiva frente a la educación, se inicio por la cobertura, pues se reconocía que existían pocas escuelas y centros educativo lo que imposibilitaba que todos los niños y niñas accedieran a la educación, pero un factor que no se tuvo en cuenta es encontrar una mediación en esa cobertura y en la calidad, pues como ellos mismos dicen "la cobertura se construyo a costa de la calidad". Siendo esto un obstáculo para las prácticas pedagógica, dejando poco espacio a la flexibilidad en el ámbito educativo.

Lo que sugiere la idea de hacer una recopilación de los antecedentes en la educación y un paralelo a lo que hoy se vive, y de esta manera crear espacios de reflexión en donde se re-signifique la calidad educativa. En primer momento la educación estaba bajo parámetros en donde la práctica docente se disponía por vocación, hoy en día está estructura es más una práctica de profesionales, en donde anteriormente la calidad la regulaba el docente bajo otros factores, en donde la comunidad educativa estaba más comprometida, en donde la cantidad de estudiantes era aceptable para la infraestructura que había, a diferencia de hoy en día en donde hay más estudiantes dentro de inadecuadas aulas con relación a la cantidad de educandos, la familia en sus múltiples ocupaciones se a distanciado del procesos pedagógico de sus hijos, entre otros.

Todos estos son factores que hacen que en la educación no se evidencie en un buen nivel la calidad, por esa razón es importante que maestros e intelectuales desde las aulas creen la resistencia bus- 
cando establecer espacios reflexivos dentro de las mismas. Es de reconocer que el Estado tiene el control en la educación ya que ejerce un poder sobre esta y muchas veces obstaculiza la opción que maestros e intelectuales puedan comprender el contexto de forma reflexiva. Pero es de anotar que esta es la oportunidad para que maestros e intelectuales movilicen la teoría en función de las prácticas, que los contenidos sean un pretexto para que ellos desde el campo conceptual movilicen las competencias y ordenen sus prácticas teniendo en cuenta al sujeto y sus metas de aprendizaje.

Aunque el Estado no proporciona los recursos necesarios para dicha transformación educativa, y algunas veces solo crea proyectos a corto tiempo ejecutados muchas veces solo como cumplimiento y que además algunos no son funcionales a determinados contextos institucionales.

Es de reconocer que envían una "carta de navegación" que es el PEI, aunque este no es orientado para ser trabajado desde una lógica reflexiva, sino desde una lógica disciplinar, lo que lleva con dificultad a muchas instituciones a tener en cuenta la subjetividad de los educandos en el currículo, a la falta de reconocimiento del contexto y ecosistemas, es de reconocer que los maestros e intelectuales desde sus fortalezas pueden movilizar desde las aulas y así contribuir a trabajar desde los intereses de los estudiantes mediados por la ciencia, en donde la ciencia este contextualizada y en donde los educandos le encuentren sentido a la educación.

Lo anterior podría encaminar las relaciones de poder que existen dentro de la escuela hacia un mismo fin, pues estas no son un arma para hacer cumplir órdenes enfatizadas a una ligera sistematización sintomática de las instituciones.

Pues estas relaciones de poder podrían ser de alguna manera las que organizan el dispositivo escolar, donde este no sea tan vertical, pues de la misma manera, tanto los docentes, como los estudiantes, como el rector y la familia se vinculan por medio de relaciones de poder que permiten mediar o no las circunstancias que se presentan dentro del aula.

Reconociendo que los contextos están atravesados por las relaciones de poder el Estado corresponde a permitir a las instituciones la posibilidad de crear espacios de reflexión en donde la educación reconozca al sujeto desde un trabajo en colectividad, buscando una construcción de proyectos vitales, de humanidad, de tejido social desde lo cotidiano en el trascurrir de cada día en las aulas.

Esto se puede iniciar desde la construcción de un currículo que pueda comprender el mundo desde los procesos de aprendizajes, desde esas lógicas disciplinares, en donde el Estado brinde la posibilidad en el que maestros e intelectuales puedan cuestionar su poder político empoderándose como sujetos, no bajo la estructura de cohesión de una evaluación por desempeños sino desde su subjetividad como referente de realidad hacia los educandos, como sujeto histórico en donde desde sus prácticas pueda resistirse y ser emancipador frente a la crisis del sin sentido que se vive en las aulas de clase.

\section{Sendero a recorrer}

Estableciendo que los jóvenes son prioridad en el futuro de la nación y lo que concierne a ellos es realidad compartida maestros e intelectuales como dice Zemelman (1998) "traspasar los límites para abrirse a lo inédito supone una necesidad de realidad que obliga a colocarse como sujetos pensantes por sobre los contenidos acumulados", en donde se crean espacios de reflexión para establecer posibilidades de mundo desde el aula.

Para ello es importante "Volver a tejer desde lo cotidiano" en donde se proponga desde el aula una construcción de proyectos vitales, de mundo, de humanidad; desde los horizontes proporcionados, desde la estructura teórica en el caso de la 
educación los estándares, para que estos sean comprendidos en un contexto y se puedan movilizar competencias, en donde la teoría movilice la práctica pedagógica.

Para esto es importante que el maestro e intelectual vuelva a ser "una voz crítica, con autoridad moral, capaz de reflexionar y hacer propuestas originales y solventes sobre la sociedad y las circunstancias" (Entrevista Blecua, 2012) ${ }^{5}$ y como ser político desde su actuar aporte a la educación en esos espacios del día a día como los son las aulas de clase, para que desde allí se construya con los educandos resistencia y emancipación a la deshumanización y a la crisis educativa.

Es desde allí, desde el aula en donde se puede construir tejido social, espacios de reflexión, en donde el maestro e intelectual puede re- significar el saber pedagógico, en donde se tenga en cuenta la historicidad de los sujetos, el contexto, los ecosistemas, en donde se enseñe un uso critico de las TIC'S, en donde se brinde posibilidades para que los educandos construyan sus proyectos de vida.

Es a partir de la escuela que se impulsan prácticas sociales educativas que permiten gestionar el conocimiento, re significar y construir el sentido para la transformación del ser y expansión de humanidad. La escuela no es una empresa, es una institución instituyente fundadora de identidad que trabaja la condición emancipadora del ser, produce prácticas sociales, moviliza el pensamiento, forma y construye sentido de humanidad.
Por consiguiente es necesario repensar la escuela, en donde todos los actores participen en colectividad de esta construcción, buscando el sentido que para ellos tiene la educación, el estar allí con relación al otro, al contexto, pues esta sería la oportunidad de pensar la escuela críticamente y a su vez proporcionaría los espacios de reflexividad para que los educandos puedan construir sus proyectos de vida, en donde el currículo tenga en cuenta la subjetividad de los jóvenes y exista un cruce de saberes, pues es de reconocer que el "individualismo como previo al sujeto, el colectivo como búsqueda del individuo con consciencia histórica, pues, como se ha observador, en el centro de la vida hay un ser "que no se podrá llamar él ni yo, sino solamente nosotros", pues la educación debe construirse desde lo cotidiano en colectividad.

Este repensar la escuela en colectividad desde volver a tejer desde lo cotidiano es un llamado a maestros e intelectuales, ya que ellos son sujetos generadores de cambio, para que desde sus constructos teóricos y su proceder creen espacios de reflexión y como referentes de realidad construyan sentido de humanidad, como dice Guarin (2010):

Es indispensable discernir nuestro lugar de sujetos autónomos en el mundo de hoy, en nuestras instituciones y organizaciones humanas, en nuestras disciplinas y profesiones, muchas veces atrapados, confinados en ella, sin lograr trascender los roles que funcionalmente nos asigna la sociedad.

5 Blecua José Manuel. (2012). Sin pensamiento crítico. En http://cultura.elpais.com/cultura/2012/02/22/actualidad/1329922234_215883. html (Recuperado el 07 de julio del 2012) 


\section{Bibliografia}

Arango Álzate, Oscar Albeiro - Meza Rueda, José Luis. (2002) El discernimiento y el proyecto de vida, Dinamismo para la construcción de sentido, Pontificia Universidad Javeriana: Ecoe ediciones.

Arendt Hannah. (1993). La construcción humana. Barcelona: Paidós.

Ball Stephen J,(1994). Foucault y la educación, Disciplinas y saber. Madrid: Ediciones Morata.

Barthes Roland. (1995). Lo obvio y lo obtuso. Barcelona: Edición Paidós.

Bourdieu Pierre. (2002). Pensamiento y acción. Buenos Aires: Libros del zorzal.

Freire Paulo. (1997). Pedagogía de la autonomía. Buenos Aires: Siglo veintiuno

Freire Paulo. (2005). Pedagogía de la esperanza, Un reencuentro con la Pedagogía del oprimido. Argentina: Siglo XXI Editores. Primera edición.

Foucault Michaell. (1991). Saber y verdad. Madrid: Editorial La Piqueta.

Foucault Michaell. (1992). Microfísica del poder. Madrid: Editorial La Piqueta.

Hanneth Axel. (2009). Patologías de la razón y actualidad de la teoría crítica. Buenos Aires: Katz editores.

García Márquez, Gabriel. (2010). Yo no vengo a decir un discurso. Barcelona: Editorial Mordadom.

Gil Serna, Marien Alexandra. (2009). Subjetividad: un tejido para construir. Manizales: Plumillas Universidad de Manizales.

González, González Miguel Alberto. (2009). Horizontes Humanos: Límites y paisajes. Manizales: Universidad de Manizales.

González, González Miguel Alberto. (2010). Umbrales de indolencia: educación sombría y justicia indiferente. Manizales: Universidad de Manizales.

Guarín Jurado, Germán. (2010). Seminario de teoría de pensamiento crítico en la educación. Manizales: Universidad de Manizales

León, Emma. Zemelman, Hugo. (1997). Hacia una sociología del sujeto, democracia y sociedad civil. Barcelona: Editorial Anthropos.

Olaya Amaya Alfredo, Sánchez Ramírez Mario, Acebedo Juan Carlos. (2001). La Tatacoa,
Ecosistema Estratégico de Colombia. Neiva - Huila: Ed. Universidad Surcolombiana. Dirección General de Investigaciones. Primera edición.

Ospina William. (2008). La escuela de la Noche. Bogotá: Grupo Editorial Norma.

Philippe Meirieu. (1998). Frankestein educador. Barcelona: Editorial Laertes.

Quintar, Estela B. (1998). La enseñanza como puente a la vida. México: Instituto politécnico nacional.

Sabato Enesto. (2001). Apologías y rechazos. Buenos Aires: Seix Barral.

Sabato Ernesto. (2000). La resistencia. Buenos Aires: Seix Barral.

Teller Janne. (2006). Nada. Barcelona: Seix Barral Biblioteca furtiva.

Zemelman Hugo. (1998). Sujeto: existencia y potencia. México: Editorial Anthropos.

Zemelman Hugo. (1998). El ángel de la historia: determinación y autonomía de la condición humana. México: Editorial Anthropos.

Blecua José Manuel. (2012). Sin pensamiento crítico. En http://cultura.elpais. com/cultura/2012/02/22/actuali dad/1329922234_215883.html (Recuperado el 07 de julio del 2012)

Corral Milagros. (2012). Sin pensamiento crítico. En http://cultura.elpais.com/cultura/2012/02/22/ actualidad/1329922234_21588.html (Recuperado el 07 de julio del 2012)

Dinvinsky Daniel. (2012). Sin pensamiento crítico. En http://cultura.elpais. com/cultura/2012/02/22/actualidad/1329922234_215883.html (Recuperado el 07 de julio del 2012)

Méndez Gallardo Mariana. (2007). En http:// www.fum.edu.co/snies/inst/Unidades/ udt/informe_web/Aprendizaje $\% 20 \mathrm{e} \% 20$ interacci\%C3\%B3n\%20social.pdf

Volpi Jorge. (2012). Sin pensamiento crítico. En http://cultura.elpais.com/cultura/2012/02/22/ actualidad/1329922234_215883.html (Recuperado el 07 de julio del 2012)

Zemelman Hugo. (2007). En: http://biblioteca. itam.mx/estudios/60-89/86/BMarianaMendezGallardoHugo.pdf (Recuperado el 09 de julio del 2012) 